functional disturbance of the glands, or whether there is actually some anatomic alteration, is not known. I have been entirely unable to obtain portions of the stomach from a fatal case of lead poisoning in order to determine this point, but the opportunity will certainly arise in the course of time.

The only conclusions that are at present justifiable appear to be:

1. In a series of 12 cases of lead poisoning, or suspected lead poisoning, there was deficiency in the secretion of $\mathrm{HCl}$ in 10 of the chronic cases, and no deficiency in 2 , one of which was doubtful and the other acute.

2. This deficiency in the secretion of free $\mathrm{HCl}$ in the majority of cases is associated with an extreme reduction in the percentage of peptic digestion, and with the presence of lactic acid.

3. It is not justifiable at present to regard it as an indication for treatment, at least not until the effects of the ordinary treatment for achylia gastrica in cases of lead poisoning have been tested.

\section{BRAIN HEMORRHAGE.*}

WILLIAM A. DICKEY, A.M, M.D.

Professor of Practice of Medicine and Clinical Medicine. Toledo Medical College; Medical Department, Toledo University; Attending Physician St. Vincent's IIospital. TOLEDO, OIIIO.

While brain hemorrhage is not of common occurrence, it happens with sufficient frequency to make its discussion of more than passing interest to the general practitioner. In this paper $\overline{\mathrm{I}}$ shall take into consideration only those cases which are the result of degeneration of the arterial tunics.

Degenerative changes in the coats of the blood ressels were noticed and commented on in the early days of medicine by Lobstein, who gave it the name by which it has since been known-arteriosclerosis. Morgagni and Senac also speak of it. To Rokitansky and Virchow and, more recently, to Thoma we are indebted for a thorough investigation of the nature of the process, which I shall not take the time to discuss. I shall assume that the pathologic changes in arteriosclerosis and atheroma are practically the same-differing only in degree. In the small arteries of the brain substance we find minute aneurismal pouches caused by the gradual giving way of the diseased coats. As these blood vessels possess no true outer coat, their dilatation under the combined influence of disease and increased blood pressure becomes an easy one. Of capillary hemorrhages following the plugging of a vein I shall not speak, and while hemorrhage from a vein may occur, hemorrhage is practically always from an artery. It will not be amiss, I think, but profitable to notice at the outset some of the causes that lead to changes in the coats of the blood vessels. These alterations may result from a multiplicity of conditions, some of which are fairly well understood, while others are decidedly obscure. When we think of arterial degeneration, we are too prone, I fear, to picture to ourselves an old man who has lived his allotted time on earth and in whom decay and deterioration are plainly visible in every body cell. "This is a fatal error. Arterial degeneration mav and often does take place, from causes we can not well fathom, in those who are comparatively young; and that it does

* Read at a meeting of the Northwestern Obio Medical Association at Tiffin, 1904 . occur in these cases is patent to all who have had a fairly wide clinical experience.

That the consumption of large quantities of alcoholic beverages is a potent factor in bringing about this degeneration has been recognized for years. It is true that not all who use alcohol in its various forms have disease of the arterial coats, but it is found too frequently in this class of cases to be more than a mere coincidence. Not only does this poison affect the blood ressels, particularly of the brain, but also, in consequence of the effect of the alcohol on the glands and mucous membrane of the stomach and liver, poisons of varying degrees of intensity and non-oxidizable in character are found circulating in the body fluids, and we have this secondary cause added to the primary, with greater or less disease in the coats of the blood vessels.

I am satisfied that among business men, weighted with the responsibilities of large enterprises, who think long and persistently and yet drink constantly, these phenomena are most marked and come on earliest. At the last session of the American Medical Association, Cabot ${ }^{1}$ of Boston read a paper in which he brought forward statistics to prove that alcohol is not the potent factor in the production of arterial degeneration that it is supposed to be; and yet the experience of the profession as a whole lends color to the belief that alcohol is, both directly and indirectly, a cause of this condition. Indeed, those who do not use alcohol in any of its forms but who lead a strenuous life, with but little freedom from worry and anxiety and with practically no exercise, become prematurely old and have premature vascular decay. In these cases, in some mysterious way. chemic or otherwise, toxins are developed which slowly but nevertheless surely bring about arterial degeneration. Or it may be, as has been suggested by Romberg, that the irregular blood pressure caused by an unstable and overwrought nervous system is the cause.

Next to alcohol as a factor in the production of these pathologic changes stands syphilis, and like alcohol its evil effects are seen in a most pronounced way in the blood paths of the brain. The syphilitic poison, whatever it may be, interferes with the nutrition of the walls of the blood vessels, destroying in a measure their elasticity, and thereby allowing them to become weakened and dilated. In some cases we may find both alcohol and syphilis operating as causes, as in a case I have under observation now.

In brain hemorrhage, chronic interstitial nephritis with its unknown antecedents should always be thought of and looked for, and is next in importance to the causes I have already assigned. In this disease, as prominent symptoms there are marked changes in the walls of the blood vessels, increased peripheral tension, followed by cardiac hypertrophy with its augmented potential energy. Whether the degeneration of the blood vessels is primary and the kidney lesion secondary or vice versa, I can not say. It is certain that toxic substances circulating through the blood and not eliminated by the kidneys, must sooner or later produce this form of degeneration. Indeed, these poisons bring about destructive changes in the kidneys in the same manner as in the coats of the arteries.

It is within the range of possibility that an increased activity on the part of the suprarenal glands may be a cause of greater or less intensity in the production of arteriosclerosis. Just to what extent this is operative as a cause can not be definitely stated, as it will require

1. The Joursal, Sept. 17, 1904, p. 774. 
a great deal of experimental, as well as clinical, rescarch to determine it.

A multiplicity of other factors might be mentioned as leading to vascular decay, but as these are sufficient for the purpose of this paper I will not consume time in their discussion. Aside from the disease of the blood vessels, another factor is essential to the production of cerebral hemorrhage, and that is increased intracranial blood pressure. Branches of the middle meningeal artery are the ones most frequently ruptured; and particularly is this true of the lenticulostriate artery. Mendel has pointed out that the normal blood pressure is higher in this artery than it is in the cortical or other small arteries of the brain. If this be true, it will account in a rational way for the more frequent rupture of this artery.

There are so many causes that lead to increased blood pressure that it would be impossible to mention them all. Indeed, the patient is seldom seen until after the artery has been ruptured. If the physician is consulted before actual mischief has been done, he should point out the danger arising from sudden, severe, muscular effort, such as lifting, running to catch street cars, straining at stool, in short, anything that is liable to excite the heart's action. I wish to emphasize particularly the injurious influence arising from the pleasures of the table. Attacks of indigestion, which in this class of cases are so liable to follow a full meal, are a very great source of danger. The heavy meal should be at midday and the last meal should be taken at least three hours before going to bed. All articles of diet which produce flatulence should be avoided because of the danger of bringing on disturbed heart action. This distention of the stomach and bowels with resulting increased cardiac force, will explain many of those cases of sudden death which are encountered at times. The individual will go to bed at a seasonable hour after a hearty meal, apparently in the best of health and with perfect tranquillity and will be found dead in the morning. Tea and coffee should be entirely omitted from the dietary for the reason that they, too, disturb cardiac repose. For the same reason, anything containing alcohol should be tabooed. There can be no doubt that as man grows older less food should be eaten, particularly meats. This applies with greater force to those who have the responsibilities of business and professional work. We are a nation of high livers, and sooner or later we must pay the penalty.

As I have already said, we are seldom called until after the hemorrhage has taken place. These bleedings are not always profuse, but at times are slight in character and well-directed management will be of incalculable benefit to the patient. Nowhere in the whole domain of medicine is it more necessary to have welldefined ideas of what to do, and then do it. In these cases we must act quickly. Delays are dangerous. The individual should be subjected to as little handling as possible. This is a matter of vital importance. If at home, he should be made comfortable just where the attack comes on him. The head should be elevated and the clothing loosened. If the extremities are cold they should be surrounded with bottles of hot water. The dosing of the patient with ergot, iron, tannic acid and bromid is worse than useless. We have two factors to contend with-a broken blood ressel and a rapidly working, forceful heart pumping blood into it.

Manifestly, then, what is needed is quickly acting, powerful cardiac depressants, something that will limit the ability of the heart to pour blood into the bleeding ressel. I need hardly say that we can not stop the flow of blood into the ruptured artery, but we can lessen it. Moreover, there is increased peripheral tension, which must be relieved. The remedies to be used, named in the order of importance, are aconite, veratrum viride and gelsemium, and venesection. If the fluid extract of aconite is used it should be given in good-sized doses until its physiologic effects are apparent, say from five to ten minims every 30 minutes, when the dose is to be lessened or the interval lengthened. In the more pronounced forms of the trouble, when the patient can not swallow well, aconitin, in $1 / 50$ or $1 / 100$ of a grain, should be given hypodermically and repeated when necessary. When veratrum viride is used the initial dose of the fluid extract should not exceed five minims. This drug is a powerful cardiac depressant, but its action should be carefully watched in order to avoid producing nausea and vomiting. Gelsemium, by its direct action on the heart. is a most serviceable drug in these cases, and because of its lessened liability to produce nausea it is possibly to be preferred to veratrum. When the face is markedly cyanosed and when the individual is decidedly fullblooded, phlebotomy may be practiced with most gratifying results; but when the individual is of spare habit the loss of sufficient blood to be beneficial might put him at a disadvantage in his future struggle for recovery. Hypodermoclysis, by reason of its causing increased cardiac action, is not to be thought of. There is a growing belicf that gelatin, by reason of its increasing the coagulability of the blood, is indicated in cases of concealed hemorrhage. It may be given by the mouth or injected under the skin. The amount hypodermically is usually about 250 c.c. of a 1 per cent. solution in a normal saline mixture.

To the larger portion of the profession, however, this procedure is lacking in practicability because gelatin might not be at hand from which to prepare the solution. Another objection is its slowness of action, and this I consider a serious one, for in cases of this character a prompt, energetic response is required. It goes without saying that stimulation with whiskey, brandy or strychnia is contraindicated.

Cathartics of any lind should not be used for the first few days because of the disturbance the act of defecation produces. When a cathartic is given, I think the mild chlorid of mercury the best.

The ice-cap to the head is recommended by competent physicians. and yet I regard it as of doubtful utility. I am at a loss to know how it can in any way influence the circulation in the deep structures of the brain, particularly such small arteries as those that are ruptured.

The patient should be kept absolutely quiet in bed for ten dars or two weeks. and during this time hould not be allowed to see any one except the physician and those in immediate attendance.

Clear-Cut Therapeutics.-One of the fatal mistakes made by the unthinking physician is to so medicate as to mask symptoms-a veritable burying of clean-cut therapeutic possibilities in medicines not indicated. This is a direct outgrowth of uncertain remedies, always the precursor of uncertain application. Given true remedies, there is courage for the physician to study certainty of application, out of both of which comes scientific practice.-Alkaloidal Clinic. 